\title{
Evoluindo do CMMI-SW Nível 3 para o CMMI-DEV Nível 5: A Experiência do Atlântico
}

\author{
Luciana Ferreira Trindade, Carla Ilane M. Bezerra, Gabi Telles, Carlo Giovano \\ Pires, Luiz Sérgio Plácido, Cicília Moreira, Joice Barbosa, Paula Luciana Ferreira. \\ Instituto Atlântico, Rua Chico Lemos, 946 \\ Caixa Postal 60822-780, Fortaleza - CE - Brasil \\ \{luciana, carlailane, gabi, cgiovano, sergio_luiz, moreira_cicilia, \\ barbosa_joice, paula\}eatlantico.com.br
}

\begin{abstract}
This work presents the planning, definition and implementation of CMMI-DEV level 5 at Atlantico. This case has been planned using the PDCA method and its implementation has been based on the Six Sigma methodology. The main results of this initiative were the enhancement of process maturity, the implementation of technological and process innovations, better estimates and a more precise control of projects' critical factors such as productivity and quality. The difficulties faced in this effort and the lessons learned are also described.
\end{abstract}

Resumo. Este trabalho relata a experiência de planejamento, definição e implantação do CMMI-DEV nível 5 no Atlântico. Esta experiência foi planejada com base no método de gerenciamento de melhoria de processos PDCA e realizada com o uso da metodologia Six Sigma. Como principais resultados obtidos, ressaltamos o amadurecimento do processo de desenvolvimento da instituição, a implementação de inovações tecnológicas de processo, a melhoria das estimativas e o maior controle de aspectos críticos dos projetos como produtividade e qualidade. São também descritas as principais dificuldades encontradas e lições aprendidas.

\section{Introdução}

O Atlântico é uma instituição de pesquisa e desenvolvimento localizada em Fortaleza, com filiais em Sobral e São Paulo, e conta com aproximadamente 270 colaboradores. Desde a sua fundação, o Atlântico iniciou um amplo programa de qualidade, aderente à norma ISO 9001 e o nível 5 do modelo CMMI-DEV 1.2 [Chrissis et al. 2006].

Neste contexto, o artigo tem como objetivo relatar a experiência do Atlântico nas atividades de planejamento, definição, institucionalização e as metodologias utilizadas para evolução do nível 3 para os níveis 4 e 5 do modelo CMMI-DEV.

Este artigo está estruturado em cinco seções. A seção 2 apresenta a motivação de evolução para o nível 5 do CMMI, a seção 3 descreve o processo de implantação, na seção 4, são apresentados resultados e lições aprendidas e a seção 5 discorre sobre a conclusão do trabalho. 


\section{Motivação para Evoluir do Nível 3 para o Nível 5 do CMMI-DEV}

Organizações que pretendem atingir os altos níveis de maturidade tem como principais motivações um visão quantitativa do desempenho de seus processos e sua melhoria contínua.

Nos níveis 4 e 5, os projetos da empresa passam a ser gerenciados quantitativamente, mediante a análise dos dados de desempenho dos processos, dos limites de controle de baseline e de modelos de desempenho. O objetivo da melhoria de processos, nestes níveis, é garantir a previsibilidade de desempenho dos processos selecionados ${ }^{1}$, removendo as causas atribuíveis (especiais), que impedem a estabilidade desses processos ${ }^{2}$, além disso, os processos da organização devem ser otimizados por meio de alterações e adaptações incrementais e inovadoras para efetivamente atender aos objetivos de negócio atuais e projetados.

\section{O Processo de Implantação do CMMI-DEV Níveis 4 e 5}

O processo de implantação do CMMI-DEV dos níveis 4 e 5 começou a ser planejado logo após a implantação do nível 3 em 2006. Desta forma, toda a estrutura organizacional estava preparada para receber as alterações necessárias nos processos para aderência aos níveis 4 e 5 . As atividades foram realizadas utilizando um método gerencial para promoção da melhoria continua, denominado ciclo PDCA [Aguiar 2002]: (P) planejamento das atividades; (D) execução das atividades; (C) verificação e controle do planejamento; (A) implantação dos processos definidos e atuar corretivamente se necessário. $O$ processo de implantação seguiu as etapas definidas na Figura 1.

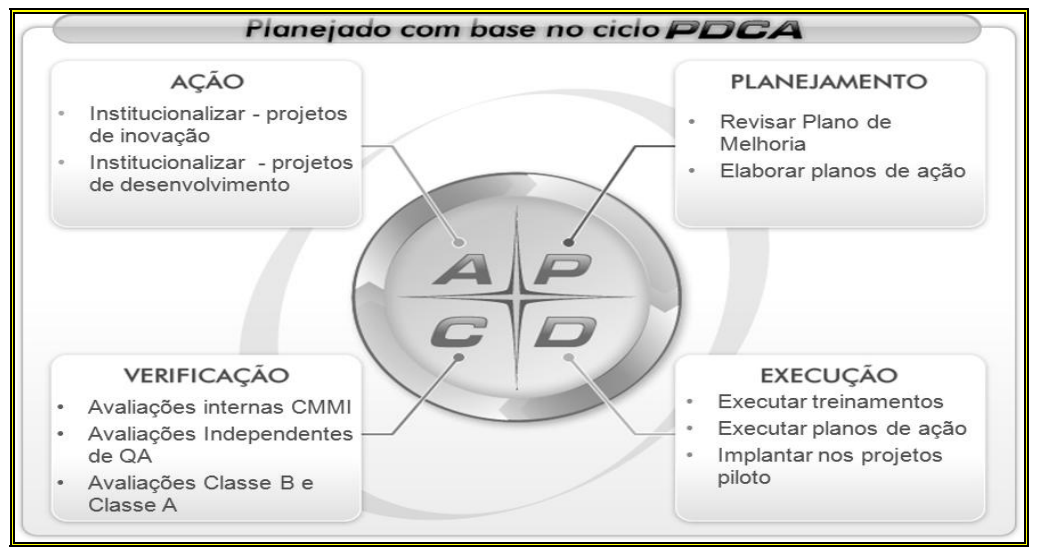

Figura 1. Etapas do Ciclo PDCA.

\subsection{O Planejamento das Atividades}

O planejamento e a gerência das atividades de melhoria dos processos do Atlântico foram de responsabilidade do grupo de Quality Assurance (QA), que contou com o suporte da consultoria de uma empresa externa certificada pelo SEI especializada em CMMI. O grupo de Quality Assurance (QA) é um grupo institucional com atuação nas

\footnotetext{
${ }^{1}$ No nível 4 são selecionados alguns processos críticos para terem seu desempenho controlado e serem gerenciados quantitativamente.

${ }^{2}$ No nível 5 o processo está sob controle, ou seja, o conjunto dos itens produzidos possui distribuição normal e essa distribuição permanece estabilizada.
} 
áreas de melhoria de processos (Engineery Process Group - EPG), qualidade, medição e análise, estatística, treinamento e gestão de configuração.

O plano de melhoria foi revisado em 2007, alinhado aos objetivos definidos no Balanced Scorecard (BSC) [Kaplan 2004], e teve como principal objetivo a implantação dos altos níveis de maturidade do CMMI-DEV. A estratégia adotada foi disparar cinco planos de ação, sendo quatro para as áreas de processo dos níveis 4 e 5 do CMMI-DEV, e um para evoluir a arquitetura de processos cobrindo a aderência à versão 1.2 do CMMI-DEV. Os planos de ação foram planejados na etapa de planejamento e utilizados na etapa de Execução. O cronograma contendo as principais atividades para implantação dos níveis 4 e 5 do CMMI-DEV é apresentado na Tabela 1.

Tabela 1. Cronograma de Implantação.

\begin{tabular}{|c|c|c|c|c|c|c|c|c|c|c|c|c|c|}
\hline \multirow{2}{*}{ Etapa } & \multirow{2}{*}{$\begin{array}{c}\text { Ano } \\
\text { Atividâdes }\end{array}$} & \multirow{2}{*}{2006} & \multicolumn{4}{|c|}{2007} & \multicolumn{4}{|c|}{2008} & \multicolumn{3}{|c|}{2009} \\
\hline & & & $1^{\mathrm{a}}$ & $2^{a}$ & $3^{\mathrm{a}}$ & $4^{\mathrm{a}}$ & $1^{a}$ & $2^{\mathrm{a}}$ & $3^{\mathrm{a}}$ & $4^{\mathrm{a}}$ & $1^{\mathrm{a}}$ & $2^{a}$ & $3^{\mathrm{a}}$ \\
\hline \multirow{2}{*}{ Planejament } & Plano de Melhoria & & & & & & & & & & & & \\
\hline & Elaborar planos de ação & & & & & & & & & & & & \\
\hline \multirow{3}{*}{ Execução } & \begin{tabular}{|l|} 
Definição e Revisão dos \\
Processos
\end{tabular} & & & & & & & & & & & & \\
\hline & Treinamentos & & & & & & & & & & & & \\
\hline & \begin{tabular}{|l|}
$\begin{array}{l}\text { Disponibilização e } \\
\text { implantação }\end{array}$ \\
\end{tabular} & & & & & & & & & & & & \\
\hline \multirow{2}{*}{ Verificação } & Avaliações Internas & & & & & & & & & & & & \\
\hline & Avaliações Externas & & & & & & & & & & & & \\
\hline \multirow{2}{*}{ Ação } & $\begin{array}{l}\text { Institucionalizar Projetos } \\
\text { de Inovação }\end{array}$ & & & & & Pilote & Pilote & Pilote & Piloto & Bilote & Org. & Org. & Org. \\
\hline & \begin{tabular}{|l|} 
Projetos de \\
Desenvolvimento
\end{tabular} & & & & & & & & & & & & Org. \\
\hline
\end{tabular}

\subsection{Definição e Revisão dos Processos}

A arquitetura de processos foi definida e documentada com base na arquitetura de processos do RUP. O RUP foi escolhido por fornecer uma notação formal para a definição de processos baseada na UML [Pires et al. 2004]. Além disso, a estrutura evolutiva e incremental presente nos processos de engenharia do RUP já era utilizada no desenvolvimento de software da organização, o que facilitou a alteração dos processos atuais e a definição dos novos processos aderentes aos níveis 4 e 5 do CMMI-DEV.

$\mathrm{Na}$ implantação dos níveis 4 e 5, além das mudanças da versão 1.2 do modelo, foram também realizadas alterações necessárias para aderência às áreas de processos dos níveis 4 e 5 do CMMI-DEV, OPP - Organizacional Process Performance, QPM Quantitative Project Management, OID - Organizational Innovation and Deployment e CAR - Causal Analysis and Resolution. Identificou-se a necessidade de novos procedimentos, orientações e templates e detectou-se o impacto das alterações em cada grupo de processos (Gestão de Projetos, Organizacionais e Suporte).

Após a alteração dos processos, o EPG realizou revisão técnica de todos os processos impactados, utilizando a técnica de Revisão pela Equipe e a abordagem de Peer Review, um método estático de verificação no qual um artefato é examinado por qualquer integrante da equipe do projeto, exceto o autor do artefato, com o propósito de detectar defeitos [Laitenberger et al. 2002]. Os processos revisados foram validados pela consultoria a fim de garantir a aderência ao modelo. 
$\mathrm{Na}$ tabela 2, temos o relacionamento entre os processos impactados e às áreas de processos dos níveis 4 e 5 .

Tabela 2. Mapeamento dos Processos Alterados e as PA's dos Níveis 4 e 5.

\begin{tabular}{||c|c|c|c||}
\hline \multirow{2}{*}{ Nivel } & PA & Grupo de Processos & Processos \\
\hline \multirow{2}{*}{4} & \multirow{2}{*}{ OPP } & Organizacional & Gestão de Processos \\
\cline { 3 - 5 } & QPM & Gestão de Projetos & $\begin{array}{c}\text { Gestão de Projetos } \\
\text { Gestão de Riscos }\end{array}$ \\
\cline { 2 - 5 } & OID & Organizacional & Gestão de Processos \\
\cline { 2 - 5 } 5 & \multirow{2}{*}{ CAR } & Organizacional & Gestão de Processos \\
\cline { 3 - 5 } & Gestão de Projetos & Gestão de Projetos \\
\hline \hline
\end{tabular}

Na figura 2, temos uma visão geral dos grupos de processos do Atlântico.

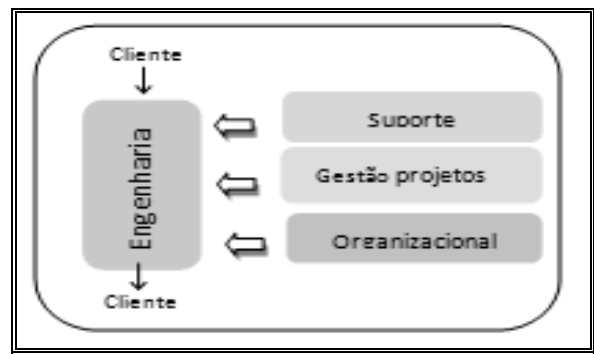

Figura 2. Visão geral dos processos da organização.

\subsection{A Disponibilização e Implantação dos Processos}

O processo de implantação dos processos alinhados ao CMMI-DEV nível 5 no Atlântico pode ser descrito em três fases. A primeira fase caracterizou-se pela realização dos treinamentos dos processos impactados. Durante esta fase, o Programa de Treinamento Organizacional foi implementado, de modo a garantir que às equipes técnica e gerencial tivessem o conhecimento e as habilidades necessárias para desempenharem seus papéis.

$\mathrm{Na}$ segunda fase iniciaram-se os ciclos de projetos de melhoria, utilizando a metodologia Six Sigma [Tayntor 2003] na implementação dos projetos DMAIC (Define, Measure, Analyse, Improve and Control) e DMADV (Define, Measure, Analyse, Design and Verify). Esta metodologia é uma estratégia que pode levar a ganhos substanciais em lucratividade e qualidade [Harry 1998]. O método DMAIC é utilizado quando um processo existente não satisfaz as necessidades ou objetivos do cliente ou apresenta desempenho abaixo do esperado [Smith e Adams 2000], enquanto o DMADV é empregado no desenvolvimento de novos produtos e processos [Carvalho et al. 2007].

Foram planejados três ciclos de projetos de melhoria. Estes projetos foram identificados a partir de idéias de inovações sugeridas pelos colaboradores ou necessidades de melhoria de desempenho relacionado aos objetivos estratégicos e foi realizada uma priorização e classificação das melhorias, conforme o método descrito em Gonçalves et al. (2008).

No primeiro ciclo, foram executados os projetos de melhoria DMAIC: Melhorar Estimativa de Produtividade e Esforço e Diminuir a Densidade de Defeitos Pré-Entrega. No segundo ciclo, foram executados os projetos de inovação DMADV, Implantar a 
Inspeção Automatizada e Implantar Processos Ágeis, e o projeto DMAIC Diminuir Retrabalho. Nestes projetos foram geradas as primeiras baselines de desempenho que posteriormente foram utilizadas para geração das primeiras versões dos modelos de previsão [Chrissis et al. 2006]. No terceiro ciclo, executamos o projeto DMAIC para Reduzir Defeitos Pós Release e o projeto DMADV para Implantar um Ambiente de Desenvolvimento de Alta Produtividade.

$\mathrm{Na}$ terceira fase iniciou a seleção dos projetos pilotos. Foram selecionados quatro projetos para a implantação dos novos processos. Os projetos selecionados representavam uma amostra significativa dos projetos existentes na instituição com relação ao número de integrantes, porte e complexidade. Essa estratégia foi adotada com o objetivo de avaliar a implantação dos processos em projetos que exigiam o suporte de uma arquitetura de processos robusta para o desenvolvimento de software.

$\mathrm{Na}$ quarta fase, os ajustes nos processos organizacionais foram realizados com base nos resultados obtidos nos pilotos, com a participação do EPG e do grupo de QA. Foi gerada a nova baseline de processos organizacionais e os processos foram implantados em toda a organização. O grupo de QA intensificou o mentoring nos projetos esclarecendo as dúvidas da equipe do projeto, participando mais ativamente das reuniões do projeto para melhor acompanhamento da implantação dos processos nos projetos.

Algumas dificuldades foram encontradas na implantação dos processos, tanto no nível organizacional como no nível dos projetos. As principais dificuldades foram:

- Geração dos modelos de previsão visto que o número de pontos eram insuficientes para que os modelos tivessem um nível aceitável de confiabilidade estatística.

- Comprovação estatística das melhorias implantadas nos projetos nas baselines de desempenho, devido aos poucos dados gerados, visto que a maioria do dos projetos possuírem iterações grandes.

- Institucionalização dos processos de gerência quantitativa. Esses novos processos trouxeram para os gerentes de projeto um novo entendimento dos dados coletados e uma nova forma de análise para tomada de decisão no tratamento dos desvios, assim como uma nova forma de adaptação dos processos e de planejamento do projeto. O conhecimento de Controle Estatístico de Processo ${ }^{3}$ (CEP) também foi necessário para o gerenciamento do projeto de acordo com as adaptações no processo de gestão de projetos.

Estas dificuldades foram superadas, com treinamentos de conceitos básicos de estatística, CEP e mentoring intensificado do grupo de QA aos gerentes dos projetos. Além de tomar iniciativas que os projetos planejassem iterações mais curtas, aumentando assim os dados da base histórica.

Foram criadas campanhas motivacionais dentro e fora do ambiente de trabalho para que todos estivessem envolvidos, motivados e comprometidos. Durante o período de institucionalização foram lançadas quatro campanhas motivacionais:

\footnotetext{
${ }^{3} \mathrm{O}$ CEP procura reduzir a variabilidade dos processos, propiciando melhores resultados como o aumento da qualidade e a redução de custos de produção [Carvalho et al. 2005].
} 
- Primeira gincana para os projetos, com duração de 6 meses, onde o projeto com o menor percentual de não conformidades na avaliação de qualidade do mês tinha direito a um privilégio (Ex. uma folga, um churrasco, etc.).

- Duas corridas de orientação, intercaladas entre as gincanas, com a participação de todos da organização. As equipes eram diversificadas e tinham que cumprir todas as tarefas determinadas. A equipe com menor tempo gasto no percurso era a vencedora.

- Segunda gincana de projetos, com duração de quatro meses, foi similar a primeira, porém com objetivos mais arrojados tratando também o desempenho do projeto em relação à produtividade e a qualidade do produto, além do projeto vencedor do mês também era premiado o projeto vencedor do período da campanha.

As estratégias adotadas tiveram grande impacto na motivação e integração dos colaboradores, no ambiente de trabalho para alcançarmos o resultado esperado.

\subsection{As Avaliações}

Durante o processo de implantação dos níveis 4 e 5 do CMMI-DEV, o Atlântico foi submetido a diversos níveis de avaliação. Em agosto de 2008 foi realizada a avaliação Classe B. O escopo desta primeira avaliação foi verificar o grau de aderência dos processos definidos com relação às áreas de processo do modelo CMMI-DEV. Em abril de 2009 foi realizada a avaliação interna e independente QA com mentoring da consultoria, o escopo desta avaliação foi verificar o grau de institucionalização dos processos. Em Julho de 2009, foi realizada a avaliação de prontidão, esta avaliação seguiu todo o formalismo exigido pelo Standard CMMI Appraisal Method for Process Improvement (SCAMPI) que é o método utilizado para avaliações oficiais do CMMI, exigindo um grande esforço da instituição na preparação do PIID (Practice Implementation Indicator Descriptions). A montagem do PIID é o ponto crítico da avaliação, pois consiste no levantamento e mapeamento de todas as evidências necessárias para comprovar a institucionalização dos processos definidos.

Em agosto de 2009 foi realizada a avaliação Classe A. O escopo desta avaliação é verificar o atendimento de todas as práticas do modelo CMMI-DEV, exceto as práticas de SAM - Supllier Agreement Management e IPPD - Integrated Product and Process Development. Foram avaliados quatro projetos com uma equipe de nove avaliadores, sendo três pessoas externas à organização. O Atlântico foi reconhecido como uma organização CMMI-DEV nível 5, sendo todas as práticas do modelo avaliadas como totalmente implementadas.

\section{Resultados Obtidos e Lições Aprendidas}

Nos projetos, o principal diferencial do nível 3 para o 5 foi em relação ao planejamento e acompanhamento dos projetos. No nível 3, a estimativa era baseada apenas em dados históricos. Com o uso da gestão quantitativa nos projetos, toda a estimativa passou a ser realizada de acordo com objetivos de desempenho do projeto e da organização. A definição destes objetivos leva em consideração os dados históricos da organização e a utilização dos modelos de previsão. Os modelos de previsão passaram a ser ferramentas 
imprescindíveis aos gestores de projetos para garantir que os objetivos definidos seriam de fato alcançados, e também para disparar ações de tratamento para os possíveis desvios. Desta forma, tivemos um menor desvio das estimativas, um acompanhamento mais efetivo dos projetos e consequentemente melhores condições de negociação de novos projetos.

$\mathrm{Na}$ organização, o principal diferencial foi em relação à análise crítica do desempenho organizacional relacionado aos objetivos do BSC. Antes da implantação dos níveis 4 e 5, a análise dos objetivos estratégicos era simplificada, ou seja, sem comprovação dos fatores que impactavam no resultado positivo ou negativo. No nível 5, os processos estão controlados estatisticamente, as ações disparadas e as melhorias implantadas tem um efeito direto nos resultados e isto pode ser mensurado e comprovado estatisticamente. Como exemplos, no âmbito organizacional, podem citar o impacto no desempenho da produtividade e da densidade de defeitos gerados com a implantação de melhorias, a partir de dois projetos de melhoria utilizando a metodologia Six Sigma. A produtividade teve redução em $66 \%$ da variação e $13 \%$ da média e a densidade de defeitos reduziu em $59 \%$ da variação e $28 \%$ da média.

Além dos resultados obtidos, ao longo do processo de implantação dos níveis 4 e 5, foram identificados um conjunto de lições aprendidas que devem ser destacadas e estão descritas a seguir:

- A participação de um especialista da área de estatística trabalhando com a equipe de definição dos processos foi muito importante para fortalecer os conceitos estatísticos nos processos.

- A ênfase de atuação do grupo de QA como orientador na condução do processo reforça a implantação e facilita a identificação de deficiências nos processos.

- A gestão do programa de treinamento é um fator crítico. Os treinamentos foram considerados essenciais e integralmente apoiados pelo corpo gerencial, pois deram suporte às demais atividades de implantação dos processos.

- As avaliações intermediárias contribuíram para o processo de melhoria. O Atlântico passou por vários níveis de avaliações preparatórias até chegar à avaliação oficial.

- Uma base histórica confiável e muito "populada" é imprescindível para facilitar a definição dos modelos de previsão e baselines de desempenho.

- O suporte de uma empresa de consultoria especializada tem expressiva importância na condução do programa como um todo. A experiência dos consultores é decisiva em momentos críticos do processo e pode, em alguns casos, abreviar os caminhos na busca dos objetivos estratégicos de qualidade definidos pela organização.

- As campanhas motivacionais é um grande fator impulsionador para o comprometimento de toda organização, o que facilita a institucionalização dos processos. 


\section{Conclusões}

A motivação para a realização deste trabalho foi divulgar a experiência do Atlântico na evolução do grau de maturidade de seus processos e que resultou no reconhecimento do nível 5 do CMMI-DEV.

De acordo com o SEI (Software Engineering Institute) atualmente apenas seis organizações brasileiras estão neste nível de maturidade em desenvolvimento de software. Dentre estas organizações, o Atlântico se destaca por ser a primeira, no Brasil, a ser avaliada no nível 5 utilizando práticas ágeis.

Com a implantação dos níveis 4 e 5 o Atlântico pôde constatar a importância de uma organização com os altos níveis de maturidade do CMMI. Isso foi observado durante todo o processo de implantação, com as novas práticas incorporadas e os resultados obtidos. Todo esforço e investimento aplicados neste projeto foram compensados com os novos desempenhos e retornos de investimento comprovados.

\section{Referências}

Aguiar, S. (2002) "Integração das ferramentas da qualidade ao PDCA e ao programa Seis Sigma”. Belo Horizonte: Editora de Desenvolvimento Gerencial.

Carvalho, M. M. (2005) “Gestão da qualidade: teoria e casos”. Rio de Janeiro: Atlas.

Carvalho, M. M., Ho, L. L., Pinto, S. H. B. (2007) "Implementação e Difusão do Programa Seis Sigma no Brasil”, Produção, v.17, n.3, p.486-501.

Gonçalves, F. M. S. , Bezerra, C. I. M., Belchior, A. D., Coelho, C. C., Pires, C. G. S. (2008) "A Strategy for Identifying, Classifying and Prioritizing Improvement and Innovation Actions: A CMMI Level 5 and Six Sigma Approach”. ASWEC.

Harry, M. J. (1998) "Six Sigma: a breakthrough strategy for profitability". Quality Process, p.60-65.

Kaplan, R. e Norton, D. A. (2004) "Strategy Maps: Converting Intangible Assets into Tangible Outcomes", Harvard Business School Press.

Kulpa, M. K., Johnson, K. A. (2003) "Interpreting the CMMI -. A process improvement approach", CRC Press LLC.

Chrissis, M., Konrad, M., Shrum, S. (2006) "CMMI: Guidelines for Process Integration and Product Improvement”, 2nd edition, Boston, Addison Wesley.

Laitenberger, O., Vegas, S., Ciolkowoski, M. (2002) "The State of the Practice of Review and Inspection Technologies in Germany", Tech Report Number: $\mathrm{ViSEK} / 011 / \mathrm{E}$.

Pires, C. G., Marinho F. G., Souza, G. T., SAMPAIO, M. (2004) "Uma Arquitetura de Processos para ISO 9001:2000 e SW-CMM Nível 3”. VI Simpósio Internacional de Melhoria de Processo de Software - SIMPROS. São Paulo.

Smith, B., Adams, E. (2000) "Lean Sigma: advanced quality". In: Proceeding of the Annual Quality Congress of the American Society for Quality, 54, Indianapolis, Indiana.

Tayntor, C. B. (2003) “Six Sigma Software Development”. Flórida, Auerbach. 